\title{
Dynamical systems of finite-dimensional metric spaces and zero-dimensional covers
}

\author{
Yuki Ikegami, Hisao Kato and Akihide Ueda \\ Institute of Mathematics, University of Tsukuba, Ibaraki 305-8571, Japan
}

\begin{abstract}
In this paper, we assume that dimensions mean the large inductive dimension Ind and the covering dimension dim. It is well-known that Ind $X=\operatorname{dim} X$ for each metric space $X$. In [7], J. Kulesza proved the theorem that every compact metric $n$ dimensional dynamical system with zero-dimensional set of periodic points can be covered by a compact metric zero-dimensional dynamical system via an at most $(n+1)^{n}$-to-one map. In this paper, we generalize Kulesza's theorem above to the case of arbitrary metric spaces, and improve the theorem. In fact, we prove that every metric $n$-dimensional dynamical system with zero-dimensional set of periodic points can be covered by a metric zero-dimensional dynamical system via an at most $2^{n}$-to-one closed map. Moreover, we also study periodic dynamical systems. We show that each finite-dimensional periodic dynamical system can be covered by a zero-dimensional periodic dynamical system via a finite-to-one closed onto map.
\end{abstract}

\section{Introduction}

In this paper, we assume that all spaces are metric spaces and dimensions mean the large inductive dimension Ind and the covering dimension dim. It is well-known that Ind $X=\operatorname{dim} X$ for each metric space $X$ (see [3, The Katětov-Morita Theorem 4.1.3]). Note that in this paper we do not use the small inductive dimension ind. We know that if $X$ is a separable metric space, then Ind $X=\operatorname{dim} X=$ ind $X$ (see [3]). A pair $(X, f)$ is called a dynamical system if $X$ is a metric space and $f$ is a homeomorphism of $X$ onto itself. A dynamical system $(Y, g)$ covers $(X, f)$ via an onto map $p: Y \rightarrow X$ provided that $p \circ g=f \circ p$. There is a classical theorem by W. Hurewicz [4] that a compact metric space $X$ is at most $n$-dimensional if and only if there is a compact metric zero-dimensional space $Z$ with an onto map $p: Z \rightarrow X$ whose fibers have cardinality at most $n+1$. Moreover, K. Kuratowski [8] and K. Morita [12] generalized this theorem to the case of arbitrary metric spaces. In the theory of dynamical systems, there is a related problem: Is every finite-dimensional dynamical system covered by a zero-dimensional dynamical system via a finite-to-one closed map? It is well known that for any compact metric dynamical system $(X, f)$, there is a compact zero-dimensional dynamical system covering $(X, f)$ via an onto map. In [7], J. Kulesza proved the following theorem: For each compact $n$-dimensional

\footnotetext{
${ }^{1} 2010$ Mathematics Subject Classification: Primary 54H20, 54F45; Secondary 37B10, 55M10, 54 C05.

${ }^{2}$ Key words and phrases: Dynamical systems, dimension, periodic points, zero-dimensional covers, general position
} 
dynamical system $(X, f)$ with zero-dimensional set of periodic points, there is a compact zero-dimensional dynamical system covering $(X, f)$ via an at most $(n+1)^{n}$-to-one map. He also showed that there is a compact one-dimensional dynamical system which can not be covered by a compact zero-dimensional dynamical system via a finite-to-one map (see [7]). Moreover, he [7] announced in his paper without proof that there is a zero-dimensional compact dynamical system covering $(X, f)$ via an at most $(n+1)$-to-one map. However, we could not find any articles about this fact. Covers of dynamical systems are also related to the theory of colorings. Many mathematicians have investigated the theory of colorings (e.g., see [1], [5], [9] and [11]).

In this paper, we generalize Kulesza's theorem to the case of (nonseparable) metric spaces, and improve the theorem. In fact, we prove that every metric $n$-dimensional dynamical system with zero-dimensional set of periodic points can be covered by a metric zero-dimensional dynamical system via an at most $2^{n}$-to-one closed map. In Lemmas 3.3, 3.4 and 3.5 of this paper, we are bit more careful in choosing locally finite open covers $\mathcal{C}(j)$ compared with the methods of Kulesza [7, Lemmas 3.5 and 3.7]. Also, in this paper we study periodic dynamical systems.

Recall that $\operatorname{dim} X$ denotes the covering dimension of a space $X$ and Ind $X=\operatorname{dim} X$ for each metric space $X$ (see [3], [11] and [13]). For the theory of dimention for arbitrary metric spaces, see [3] and [13]. Let $\mathbb{N}$ be the set of all natural numbers, i.e. $\mathbb{N}=$ $\{1,2,3, \ldots\}$, and let $\mathbb{Z}$ be the set of all integers, i.e. $\mathbb{Z}=\{\ldots,-2,-1,0,1,2, \ldots\}$. For each map $f: X \rightarrow X$, let $P(f)$ be the set of all periodic points of $f$, i.e.

$$
P(f)=\left\{x \in X \mid f^{j}(x)=x \text { for some } j \in \mathbb{N}\right\} .
$$

If $K$ is a subset of $X$, then $\mathrm{cl}(K), \operatorname{bd}(K)$ and $\operatorname{int}(K)$ denote the closure, the boundary and the interior of $K$ in $X$, respectively. For a collection $\mathcal{C}$ of subsets of $X,\left.\mathcal{C}\right|_{K}=\{C \cap K \mid$ $C \in \mathcal{C}\}$ and $\operatorname{ord}(\mathcal{C})=\sup \left\{\operatorname{ord}_{x} \mathcal{C} \mid x \in X\right\}$, where $\operatorname{ord}_{x} \mathcal{C}$ is the number of members of $\mathcal{C}$ which contains $x$.

Moreover, we need the following notions. A closed set $K$ in $X$ is regular closed in $X$ if $\operatorname{cl}(\operatorname{int}(K))=K$. A collection $\mathcal{C}$ of regular closed sets in $X$ is called a regular closed partition of $X$ provided that $\bigcup \mathcal{C}=X$ and $C \cap C^{\prime}=\operatorname{bd}(C) \cap \operatorname{bd}\left(C^{\prime}\right)$ for each $C, C^{\prime} \in \mathcal{C}$ with $C \neq C^{\prime}$. For regular closed partitions $\mathcal{A}$ and $\mathcal{B}$ of $X, \mathcal{A} @ \mathcal{B}$ denotes the regular closed partition

$$
\{\operatorname{cl}(\operatorname{int}(A) \cap \operatorname{int}(B)) \mid A \in \mathcal{A} \text { and } B \in \mathcal{B}\}
$$

of $X$. It is clear that $\operatorname{ord}(\mathcal{A} @ \mathcal{B}) \leq \operatorname{ord}(\mathcal{A}) \cdot \operatorname{ord}(\mathcal{B})$. A collection $\left\{A_{\lambda}\right\}_{\lambda \in \Lambda}$ of subsets of $X$ is called a swelling of a collection $\left\{B_{\lambda}\right\}_{\lambda \in \Lambda}$ of subsets of $X$ provided that $B_{\lambda} \subset A_{\lambda}$ for each $\lambda \in \Lambda$, and if $m \in \mathbb{N}$ and $\lambda_{1}, \ldots, \lambda_{m} \in \Lambda$, then

$$
\bigcap_{i=1}^{m} A_{\lambda_{i}} \neq \emptyset \text { if and only if } \bigcap_{i=1}^{m} B_{\lambda_{i}} \neq \emptyset \text {. }
$$

Conversely, a cover $\left\{B_{\lambda}\right\}_{\lambda \in \Lambda}$ of $X$ is called a shrinking of a cover $\left\{A_{\lambda}\right\}_{\lambda \in \Lambda}$ of $X$ if $B_{\lambda} \subset A_{\lambda}$ for each $\lambda \in \Lambda$.

In [7], J. Kulesza proved the following theorem. 
Theorem 1.1. ([7, Theorem 3.1]) If $(X, f)$ is a compact dynamical system such that $\operatorname{dim} X=n<\infty$ and $\operatorname{dim} P(f) \leq 0$, then $(X, f)$ can be covered by a compact zerodimensional dynamical system via an at most $(n+1)^{n}$-to-one map.

J. Kulesza [7] needed the following theorem to prove Theorem 1.1.

Theorem 1.2. ([7, Theorem 3.2]) Suppose that $(X, f)$ is a compact dynamical system with $\operatorname{dim} X=n<\infty$, and $\left\{\mathcal{C}_{i} \mid i=0,1,2, \ldots\right\}$ is a sequence of regular closed partitions of $X$ such that

(i) there is $m \in \mathbb{N}$ such that $\operatorname{ord}\left(\mathcal{C}_{i}\right) \leq m$ for each $i=0,1,2, \ldots$,

(ii) $\mathcal{C}_{i+1}$ refines $f^{-1}\left(\mathcal{C}_{i}\right) @ \mathcal{C}_{i} @ f\left(\mathcal{C}_{i}\right)$,

(iii) if $i>0$, then $\operatorname{mesh}\left(\mathcal{C}_{i}\right)<1 / i$.

Then there is a compact zero-dimensional dynamical system $(C, h)$ and an at most $m$-toone map $g: C \rightarrow X$ such that $g \circ h=f \circ g$.

On the other hand, for the case of arbitrary metric spaces, we know the following theorem which characterizes dimension, by use of closed coverings. The theorem is due to K. Morita [12].

Theorem 1.3. ([13, Theorem III.9]) A metric space $X$ is at most $n$-dimensional (i.e. $\operatorname{dim} X \leq n)$ if and only if there is a sequence $\left\{\mathcal{F}_{i} \mid i \in \mathbb{N}\right\}$ of locally finite closed coverings of $X$ such that

(i) for each $x \in X$ and for each neighborhood $U(x)$ of $x$ in $X$, there is $i \in \mathbb{N}$ satisfying $\operatorname{St}\left(x, \mathcal{F}_{i}\right) \subset U(x)$, where $\operatorname{St}\left(x, \mathcal{F}_{i}\right)=\bigcup\left\{F \in \mathcal{F}_{i} \mid x \in F\right\}$,

(ii) $\mathcal{F}_{i}=\left\{F\left(\alpha_{1}, \ldots, \alpha_{i}\right) \mid \alpha_{k} \in \Omega\right.$ and $\left.k=1, \ldots, i\right\}$, where $F\left(\alpha_{1}, \ldots, \alpha_{i}\right)$ may be empty,

(iii) $F\left(\alpha_{1}, \ldots, \alpha_{i-1}\right)=\bigcup\left\{F\left(\alpha_{1}, \ldots, \alpha_{i-1}, \beta\right) \mid \beta \in \Omega\right\}$,

(iv) $\operatorname{ord}\left(\mathcal{F}_{i}\right) \leq n+1$.

\section{Periodic dynamical systems and zero-dimensional covers}

In this section, we study the relation between periodic dynamical systems and zerodimensional covers. We need the following lemma which is a generalization of Theorem 1.2 to the case of arbitrary metric spaces. The proof is a modification of the proof of $[7$, Theorem 3.2]. For completeness, we give the proof.

Lemma 2.1. (cf. [7, Theorem 3.2]) Let $(X, f)$ be a dynamical system of a metric space $X$, and suppose that $\{\mathcal{C}(j) \mid j \in \mathbb{N}\}$ is a sequence of locally finite regular closed partitions of $X$ such that

(i) there is $m \in \mathbb{N}$ such that $\operatorname{ord}(\mathcal{C}(j)) \leq m$ for each $j \in \mathbb{N}$,

(ii) $\mathcal{C}(j+1)$ refines $f^{-1}(\mathcal{C}(j)) @ \mathcal{C}(j) @ f(\mathcal{C}(j))$,

(iii) for each $x \in X$ and for each neighborhood $U(x)$ of $x$ in $X$, there is $j \in \mathbb{N}$ satisfying $\operatorname{St}(x, \mathcal{C}(j)) \subset U(x)$.

Then there is a dynamical system $(C, \tilde{f})$ of a zero-dimensional metric space $C$ (i.e. 
$\operatorname{dim} C=0)$ and an at most $m$-to-one closed onto map $p: C \rightarrow X$ such that $p \circ \tilde{f}=f \circ p$. Moreover, if $X$ is a compact (resp.separable) metric space, then $C$ can be taken as a compact (resp.separable) metric space.

Proof. Without loss of generality, we may assume that each element of each $\mathcal{C}(j)$ is nonempty. Put

$$
C=\left\{\left(c_{1}, c_{2}, \ldots\right) \in \prod_{j \in \mathbb{N}} \mathcal{C}(j) \mid c_{1} \supset c_{2} \supset \cdots \text { and } \bigcap_{j \in \mathbb{N}} c_{j} \neq \emptyset\right\}
$$

and suppose that each $\mathcal{C}(j)$ is a discrete (metric) space, i.e., $\{c\}$ is open in $\mathcal{C}(j)$ for each $c \in \mathcal{C}(j)$. By (iii), note that $\bigcap_{j \in \mathbb{N}} c_{j}$ is a one point set for each $\left(c_{1}, c_{2}, \ldots\right) \in C$. Since the product space $\prod_{j \in \mathbb{N}} \mathcal{C}(j)$ is a zero-dimenisonal metric space, so is $C$. Moreover, if $X$ is a compact (resp. separable) metric space, we can take $\mathcal{C}(j)$ as a finite (resp. countable) collection and hence $\mathcal{C}(j)$ is a compact (resp. separable) metric space. In particular, $C$ is the limit of the inverse sequence $(\mathcal{C}(1) \leftarrow \mathcal{C}(2) \leftarrow \mathcal{C}(3) \leftarrow \cdots)$, if each $\mathcal{C}(j)$ is a compact metric space. Then $C$ is also a compact (resp. separable) metric space.

Define $p: C \rightarrow X$ by $p\left(c_{1}, c_{2}, \ldots\right)=\bigcap_{j \in \mathbb{N}} c_{j}$. It is easy to see that $p$ is a continuous onto map.

First, we will show that $p$ is at most $m$-to-one. Suppose, on the contrary, that there is $x \in X$ and pairwise distinct $m+1$ elements

$$
\left(c(1)_{1}, c(1)_{2}, \ldots\right),\left(c(2)_{1}, c(2)_{2}, \ldots\right), \ldots,\left(c(m+1)_{1}, c(m+1)_{2}, \ldots\right)
$$

of $C$ such that

$$
\bigcap_{j \in \mathbb{N}} c(1)_{j}=\cdots=\bigcap_{j \in \mathbb{N}} c(m+1)_{j}=\{x\} .
$$

For each $1 \leq i<i^{\prime} \leq m+1$, let $r_{i, i^{\prime}}=\min \left\{j \in \mathbb{N} \mid c(i)_{j} \neq c\left(i^{\prime}\right)_{j}\right\}$. Note that $c(i)_{j} \neq c\left(i^{\prime}\right)_{j}$ for each $j \geq r_{i, i^{\prime}}$. Put

$$
r=\max \left\{r_{i, i^{\prime}} \mid 1 \leq i<i^{\prime} \leq m+1\right\} .
$$

Then $c(1)_{r}, \ldots, c(m+1)_{r}$ are pairwise distinct elements of $\mathcal{C}(r)$ satisfying $x \in c(1)_{r} \cap \cdots \cap$ $c(m+1)_{r}$. Thus, $\operatorname{ord}(\mathcal{C}(r)) \geq m+1$. This is a contradiction.

Next, we will show that $p$ is a closed map. For each $n \in \mathbb{N}$ and $\left(c_{1}, c_{2}, \ldots\right) \in C$, let

$$
O_{n}\left(c_{1}, c_{2}, \ldots\right)=\left\{\left(c_{1}^{\prime}, c_{2}^{\prime}, \ldots\right) \in C \mid c_{n}^{\prime}=c_{n}\right\}
$$

Note that $\left\{O_{n}\left(c_{1}, c_{2}, \ldots\right) \mid n \in \mathbb{N}\right\}$ is a neighborhood base at $\left(c_{1}, c_{2}, \ldots\right)$ and $p\left(O_{n}\left(c_{1}, c_{2}, \ldots\right)\right)=$ $c_{n}$. Now let $K$ be closed in $C$ and $x \in X-p(K)$. Then $K$ and the finite set $p^{-1}(x)$ are disjoint closed sets in $C$. Put $O_{n}(K)=\bigcup\left\{O_{n}\left(c_{1}, c_{2}, \ldots\right) \mid\left(c_{1}, c_{2}, \ldots\right) \in K\right\} \subset C$. Then we can take $j \in \mathbb{N}$ with $p^{-1}(x) \cap O_{j}(K)=\emptyset$ and put $H_{j}=\bigcup\left\{c_{j} \mid\left(c_{1}, c_{2}, \ldots\right) \in K\right\} \subset X$. Note that $\left.x \notin p\left(O_{j}(K)\right)\right)=H_{j} \supset p(K)$. Since $\mathcal{C}(j)$ is locally finite, $H_{j}$ is a closed set in $X$. Put $U=X-H_{j}$. Then $U$ is an open neighborhood of $x$ in $X$ with $U \cap p(K)=\emptyset$. This implies that $p(K)$ is closed in $X$. 
Next, we will construct a desired homeomorphism $\tilde{f}: C \rightarrow C$. By (ii), note that both $f(\mathcal{C}(j+1))$ and $f^{-1}(\mathcal{C}(j+1))$ are refinements of $\mathcal{C}(j)$. Thus, by regular closedness there are unique maps $\tilde{f}_{j}, \tilde{g}_{j}: \mathcal{C}(j+1) \rightarrow \mathcal{C}(j)$ given by $\tilde{f}_{j}\left(c_{j+1}\right)=c_{j, f}$ if $f\left(c_{j+1}\right) \subset c_{j, f}$, and $\tilde{g}_{j}\left(c_{j+1}\right)=c_{j, f^{-1}}$ if $f^{-1}\left(c_{j+1}\right) \subset c_{j, f^{-1}}$. Now define $\tilde{f}, \tilde{g}: C \rightarrow C$ by $\tilde{f}\left(c_{1}, c_{2}, \ldots\right)=$ $\left(\tilde{f}_{1}\left(c_{2}\right), \tilde{f}_{2}\left(c_{3}\right), \ldots\right)$ and $\tilde{g}\left(c_{1}, c_{2}, \ldots\right)=\left(\tilde{g}_{1}\left(c_{2}\right), \tilde{g}_{2}\left(c_{3}\right), \ldots\right)$. We show that the following conditions (a), (b), (c) and (d) are satisfied.

(a) $\tilde{f}$ is continuous.

This is obvious since each $\tilde{f}_{j}$ is continuous.

(b) $\tilde{f}$ is bijective.

Let $\left(c_{1}, c_{2}, \ldots\right) \in C$. Then,

$$
\begin{aligned}
& \tilde{f}_{j} \circ \tilde{g}_{j+1}\left(c_{j+2}\right) \supset f \circ f^{-1}\left(c_{j+2}\right)=c_{j+2} \\
& \tilde{g}_{j} \circ \tilde{f}_{j+1}\left(c_{j+2}\right) \supset f^{-1} \circ f\left(c_{j+2}\right)=c_{j+2} .
\end{aligned}
$$

Note that $\tilde{f}_{j} \circ \tilde{g}_{j+1}\left(c_{j+2}\right)=\tilde{g}_{j} \circ \tilde{f}_{j+1}\left(c_{j+2}\right)=c_{j}$. Then, $\tilde{f} \circ \tilde{g}=\tilde{g} \circ \tilde{f}=\mathrm{id}_{C}$. Therefore $\tilde{f}$ is bijective and $\tilde{f}^{-1}=\tilde{g}$.

(c) $\tilde{f}^{-1}$ is continuous.

This is obvious since each $\tilde{g}_{j}$ is continuous.

(d) $p \circ \tilde{f}=f \circ p$.

Let $\left(c_{1}, c_{2}, \ldots\right) \in C$. Then

$$
\begin{gathered}
p \circ \tilde{f}\left(c_{1}, c_{2}, \ldots\right)=p\left(\tilde{f}_{1}\left(c_{2}\right), \tilde{f}_{2}\left(c_{3}\right), \ldots\right)=\bigcap_{j \in \mathbb{N}} \tilde{f}_{j}\left(c_{j+1}\right) \supset \bigcap_{j \in \mathbb{N}} f\left(c_{j+1}\right), \\
f \circ p\left(c_{1}, c_{2}, \ldots\right)=f\left(\bigcap_{j \in \mathbb{N}} c_{j}\right)=\bigcap_{j \in \mathbb{N}} f\left(c_{j}\right)=\bigcap_{j \in \mathbb{N}} f\left(c_{j+1}\right) .
\end{gathered}
$$

Therefore, $p \circ \tilde{f}\left(c_{1}, c_{2}, \ldots\right) \supset f \circ p\left(c_{1}, c_{2}, \ldots\right)$. Note that $p \circ \tilde{f}\left(c_{1}, c_{2}, \ldots\right)$ and $f \circ p\left(c_{1}, c_{2}, \ldots\right)$ are one point sets in $X$. Thus $p \circ \tilde{f}=f \circ p$.

In general, we have the following proposition. The proposition in the case of compact spaces is well known.

Proposition 2.2. Let $(X, f)$ be a dynamical system of a metric space $X$. Then there is a dynamical system $(C, \tilde{f})$ of a zero-dimensional metric space $C$ and a closed onto map $p: C \rightarrow X$ such that $p \circ \tilde{f}=f \circ p$. Moreover, if $X$ is a compact (resp. separable) space, then $C$ can be taken as a compact (resp. separable) space.

Proof. It is easy to see that we have a sequence $\{\mathcal{C}(j) \mid j \in \mathbb{N}\}$ of locally finite regular closed partitions of $X$ satisfying the conditions (ii) and (iii) as in Lemma 2.1. By Lemma 2.1, we have the desired homeomorphism $\tilde{f}: C \rightarrow C$ of a zero-dimensional metric space $C$ and a closed onto map $p: C \rightarrow X$.

A homeomorphism $f: X \rightarrow X$ of a space $X$ is periodic if $f^{k}=\operatorname{id}_{X}$ for some $k \in \mathbb{N}$. The following theorem says that each finite-dimensional periodic dynamical system can be covered by a zero-dimensional periodic dynamical system via a finite-to-one closed onto map. The case of $k=1$ implies the above theorem of Hurewicz. 
Theorem 2.3. Let $(X, f)$ be a dynamical system of a metric space $X$ such that $\operatorname{dim} X=$ $n<\infty$ and $f^{k}=\operatorname{id}_{X}$ for some $k \in \mathbb{N}$. Then there is a dynamical system $(C, \tilde{f})$ of a zerodimensional metric space $C$ with $\tilde{f}^{k}=\mathrm{id}_{C}$ and an at most $(n+1)^{k}$-to-one closed onto map $p: C \rightarrow X$ such that $p \circ \tilde{f}=f \circ p$. Moreover, if $X$ is a compact (resp. separable) metric space, then $C$ can be taken as a compact (resp. separable) metric space.

Proof. By Theorem 1.3, there is a sequence $\left\{\mathcal{F}_{i} \mid i \in \mathbb{N}\right\}$ of locally finite regular closed partitions of $X$ such that

(i) for each $x \in X$ and for each neighborhood $U(p)$ of $x$ in $X$ there is $i \in \mathbb{N}$ satisfying $\operatorname{St}\left(x, \mathcal{F}_{i}\right) \subset U(x)$,

(ii) $\mathcal{F}_{i}=\left\{F\left(\alpha_{1}, \ldots, \alpha_{i}\right) \mid \alpha_{k} \in \Omega\right.$ and $\left.k=1, \ldots, i\right\}$, where $F\left(\alpha_{1}, \ldots, \alpha_{i}\right)$ may be empty,

(iii) $F\left(\alpha_{1}, \ldots, \alpha_{i-1}\right)=\bigcup\left\{F\left(\alpha_{1}, \ldots, \alpha_{i-1}, \beta\right) \mid \beta \in \Omega\right\}$,

(iv) $\operatorname{ord}\left(\mathcal{F}_{i}\right) \leq n+1$.

Note that $\mathcal{F}_{i+1}$ refines $\mathcal{F}_{i}$. Put $\mathcal{C}_{i}=@_{j=0}^{k-1} f^{j}\left(\mathcal{F}_{i}\right)$. Since $f^{k}=\mathrm{id}_{X}$, we see that

$$
\mathcal{C}_{i}=@_{p=1}^{i} @_{j=-(k-1)}^{k-1} f^{j}\left(\mathcal{F}_{p}\right) .
$$

Then

$$
\operatorname{ord}\left(\mathcal{C}_{i}\right) \leq \prod_{j=0}^{k-1} \operatorname{ord}\left(f^{j}\left(\mathcal{F}_{i}\right)\right) \leq(n+1)^{k},
$$

and $\mathcal{C}_{i+1}$ refines $f^{-1}\left(\mathcal{C}_{i}\right) @ \mathcal{C}_{i} @ f\left(\mathcal{C}_{i}\right)=\mathcal{C}_{i}$. Therefore, applying Lemma 2.1, we obtain a desired homeomorphism $\tilde{f}: C \rightarrow C$ of a zero-dimensional metric space $C$ and a desired closed map $p: C \rightarrow X$. Finally, we will show that $\tilde{f}^{k}=\mathrm{id}_{C}$. For each $\left(c_{1}, c_{2}, \ldots\right) \in C$,

$$
\tilde{f}_{i} \circ \cdots \circ \tilde{f}_{i+(k-1)}\left(c_{i+k}\right) \supset f^{k}\left(c_{i+k}\right)=c_{i+k},
$$

where $\tilde{f}_{i}$ is the map as in the proof of Lemma 2.1. Note that $\tilde{f}_{i} \circ \cdots \circ \tilde{f}_{i+(k-1)}\left(c_{i+k}\right)=c_{i}$. Then

$$
\tilde{f}^{k}\left(c_{1}, c_{2}, \ldots\right)=\left(\tilde{f}_{1} \circ \cdots \circ \tilde{f}_{k}\left(c_{k+1}\right), \tilde{f}_{2} \circ \cdots \circ \tilde{f}_{k+1}\left(c_{k+2}\right), \ldots\right)=\left(c_{1}, c_{2}, \ldots\right) .
$$

Therefore, $\tilde{f}^{k}=\mathrm{id}_{C}$.

\section{Dynamical systems with zero-dimensional sets of periodic points}

In this section, we generalize Theorem 1.1 to the case of any metric spaces, and improve the theorem. The following is the main theorem in this paper.

Theorem 3.1. Let $(X, f)$ be a dynamical system of a metric space $X$ such that $\operatorname{dim} X=$ $n<\infty$ and $\operatorname{dim} P(f) \leq 0$. Then there is a dynamical system $(C, \tilde{f})$ of a zero-dimensional metric space $C$ and an at most $2^{n}$-to-one closed onto map $p: C \rightarrow X$ such that $p \circ \tilde{f}=f \circ p$. Moreover, if $X$ is a compact (resp. separable) metric space, then $C$ can be taken as a compact (resp. separable) metric space. 
Remark 1. J. Kulesza [7] showed that if we do not assume $\operatorname{dim} P(f) \leq 0$, there is a compact one-dimensional dynamical system which can not be covered by a compact zerodimensional dynamical system via a finite-to-one closed map (see [7, Example 2.2]).

We say a collection $\mathcal{G}$ of subsets of a metric space $X$ with $\operatorname{dim} X=n<\infty$ is in general position provided that if $\mathcal{S} \subset \mathcal{G}$ and $1 \leq|\mathcal{S}| \leq n+1$, then $\operatorname{dim}(\bigcap \mathcal{S}) \leq n-|\mathcal{S}|$, where $|\mathcal{S}|$ denotes the cardinality of $\mathcal{S}$. To get the degree of the (dynamical system) cover to be an improvement to $2^{n}$ from $(n+1)^{n}$, we need the next lemmas 3.2, 3.3, 3.4 and 3.5 which are the key lemmas. In [7, Lemma 3.7], Kulesza made the collection $\left\{f^{k}\left(b C_{i}\right) \mid k \in \mathbb{Z}, i \in \mathbb{N}\right\}$ having order at most $n$ (see [7] for the definition of $b C_{i}$ ). In this paper, we are able to get more sets $\left\{f^{p}\left(b d\left(C(j)_{\alpha}\right) \mid \alpha<\tau, j \in \mathbb{N}, p \in \mathbb{Z}\right\}\right.$ to have order at most $n$ (see Lemma 3.5 of this paper). The next lemmas are modifications of [7, Lemmas 3.5 and 3.7] in the case of nonseparable metric spaces, but we need more precise and general arguments.

Lemma 3.2. Let $X$ be a metric space with $\operatorname{dim} X=n<\infty$, and for any $j \in \mathbb{N}$, suppose that $\mathcal{G}(j)$ is a locally finite collection of closed subsets of $X$. If each $\mathcal{G}(j)$ is in general position, then there is a zero-dimensional $F_{\sigma}$-set $Z$ of $X$ such that if $A$ is a subset of $X$ with $A \cap Z=\emptyset$, then $\mathcal{G}(j) \cup\{A\}$ is in general position for each $j \in \mathbb{N}$.

Proof. Let $j \in \mathbb{N}$. Note that $\{\bigcap \mathcal{S} \mid \mathcal{S} \subset \mathcal{G}(j)$ and $\bigcap \mathcal{S} \neq \emptyset\}$ is a locally finite collection such that each element is a closed set in $X$. First, we choose a zero-dimensional $F_{\sigma}$-set $Z^{\prime}$ of $X$ such that $\operatorname{dim}\left(X-Z^{\prime}\right) \leq n-1$ (see [2, Proposition 3]). Also, for each $\mathcal{S} \subset \mathcal{G}(j)$ with $\bigcap \mathcal{S} \neq \emptyset$, we can choose a zero-dimensional $F_{\sigma}$-set $Z_{\mathcal{S}}$ of $\bigcap \mathcal{S}$ such that

$$
\operatorname{dim}\left(\bigcap \mathcal{S}-Z_{\mathcal{S}}\right) \leq \operatorname{dim}(\bigcap \mathcal{S})-1
$$

Note that $\left\{Z_{\mathcal{S}} \mid \mathcal{S} \subset \mathcal{G}(j)\right.$ and $\left.\bigcap \mathcal{S} \neq \emptyset\right\}$ is a locally finite collection of zero-dimensional $F_{\sigma}$-sets of $X$. Then

$$
Z=\bigcup\left\{Z_{\mathcal{S}} \mid j \in \mathbb{N}, \mathcal{S} \subset \mathcal{G}(j) \text { and } \bigcap \mathcal{S} \neq \emptyset\right\} \cup Z^{\prime}
$$

is also a zero-dimensional $F_{\sigma}$-set of $X$. We will show that $Z$ is a desired set. Now suppose $A \subset X$ with $A \cap Z=\emptyset$. Let $\mathcal{S} \subset \mathcal{G}(j) \cup\{A\}$ such that $1 \leq|\mathcal{S}| \leq n+1$ and $\cap \mathcal{S} \neq \emptyset$. We may assume that $A \in \mathcal{S}$. If $|\mathcal{S}|=1$, then

$$
\operatorname{dim}(\bigcap \mathcal{S})=\operatorname{dim} A \leq \operatorname{dim}\left(X-Z^{\prime}\right) \leq n-1
$$

On the other hand, suppose $2 \leq|\mathcal{S}| \leq n+1$. Since $\mathcal{S}-\{A\} \subset \mathcal{G}(j)$ and $1 \leq|\mathcal{S}-\{A\}| \leq n$ and $\bigcap(\mathcal{S}-\{A\}) \neq \emptyset$, we see that

$$
\begin{aligned}
\operatorname{dim}(\bigcap \mathcal{S}) & =\operatorname{dim}[\bigcap(\mathcal{S}-\{A\}) \cap A] \leq \operatorname{dim}\left[\bigcap(\mathcal{S}-\{A\})-Z_{(\mathcal{S}-\{A\})}\right] \\
& \leq \operatorname{dim}[\bigcap(\mathcal{S}-\{A\})]-1 \leq(n-|\mathcal{S}-\{A\}|)-1 \\
& =n-(|\mathcal{S}|-1)-1=n-|\mathcal{S}|
\end{aligned}
$$

Therefore $\mathcal{G}(j) \cup\{A\}$ is in general position for any $j \in \mathbb{N}$. 
From now on, we assume that $\tau$ and $\gamma$ are ordinal numbers. Also, we assume that 0 is the smallest ordinal number.

Lemma 3.3. (cf. [5, Lemma 2.1]) Let $\mathcal{C}=\left\{C_{\alpha} \mid \alpha<\tau\right\}$ be a locally finite open cover of a metric space $X$ with $\operatorname{dim} X=n<\infty$, and let $\mathcal{B}=\left\{B_{\alpha} \mid \alpha<\tau\right\}$ be a closed shrinking of $\mathcal{C}$. Suppose that $O$ is an open set of $X, Z$ is an at most zero-dimensional $F_{\sigma}$-set of $O$, and for each $j \in \mathbb{N}, \mathcal{G}(j)$ is a locally finite collection of closed subsets of $O$. If each $\mathcal{G}(j)$ is in general position, then there is an open shrinking $\mathcal{C}^{\prime}=\left\{C_{\alpha}^{\prime} \mid \alpha<\tau\right\}$ of $\mathcal{C}$ such that for each $\alpha<\tau$,

(1) $B_{\alpha} \subset C_{\alpha}^{\prime} \subset C_{\alpha}$,

(2) $C_{\alpha}^{\prime}=C_{\alpha}$ if $\operatorname{bd}\left(C_{\alpha}\right) \cap O=\emptyset$,

(3) $C_{\alpha}^{\prime}-O=C_{\alpha}-O$,

(4) $\operatorname{bd}\left(C_{\alpha}^{\prime}\right)-O \subset \operatorname{bd}\left(C_{\alpha}\right)-O$,

(5) $\operatorname{bd}\left(C_{\alpha}^{\prime}\right) \cap Z=\emptyset$,

(6) $\mathcal{G}(j) \cup\left\{\operatorname{bd}\left(C_{\alpha}^{\prime}\right) \cap O \mid \alpha<\tau\right\}$ is in general position for any $j \in \mathbb{N}$.

Proof. Without loss of generality, we may assume that $B_{0}=C_{0}=X(\alpha=0)$. We will construct $C_{\alpha}^{\prime}$ by transfinite induction on the ordinal $\alpha$. For the case $\alpha=0$, we put $C_{0}^{\prime}=X$. Next we assume that there is $\left\{C_{\beta}^{\prime} \mid \beta<\alpha\right\}(\alpha<\tau)$ satisfying the conditions (1)-(5) and

$$
\mathcal{G}(j) \cup\left\{\operatorname{bd}\left(C_{\beta}^{\prime}\right) \cap O \mid \beta<\alpha\right\}
$$

is in general position for each $j \in \mathbb{N}$. We will construct $C_{\alpha}^{\prime}$ as follows. By Lemma 3.2, there is a zero-dimensional $F_{\sigma}$-set $Z_{\alpha}$ of $O$ such that if $A \subset O$ and $A \cap Z_{\alpha}=\emptyset$, then $\mathcal{G}(j) \cup\left\{\operatorname{bd}\left(C_{\beta}^{\prime}\right) \cap O \mid \beta<\alpha\right\} \cup\{A\}$ is in general position for each $j \in \mathbb{N}$. Consider the following open subspace of $X$ :

$$
Y_{\alpha}=X-\left[\operatorname{bd}\left(C_{\alpha}\right)-O\right]
$$

Also consider the following closed set of $Y_{\alpha}$ :

$$
B_{\alpha}^{\prime}=\left[B_{\alpha} \cup\left(\operatorname{cl}\left(C_{\alpha}\right)-O\right)\right] \cap Y_{\alpha}=B_{\alpha} \cup\left(C_{\alpha}-O\right)
$$

Since $\operatorname{dim}\left(Z_{\alpha} \cup Z\right) \leq 0$, we can choose an open set $C_{\alpha}^{\prime}$ of $Y_{\alpha}$ such that $B_{\alpha}^{\prime} \subset C_{\alpha}^{\prime} \subset$ $\operatorname{cl}_{Y_{\alpha}}\left(C_{\alpha}^{\prime}\right) \subset C_{\alpha}$ and $\operatorname{bd}_{Y_{\alpha}}\left(C_{\alpha}^{\prime}\right) \cap\left(Z_{\alpha} \cup Z\right)=\emptyset$ (see [3, p.221, Theorem 4.1.13]). Then $C_{\alpha}^{\prime}$ is an open set of $X$. Note that $\operatorname{bd}_{Y_{\alpha}}\left(C_{\alpha}^{\prime}\right) \subset O$ and $\operatorname{bd}\left(C_{\alpha}^{\prime}\right) \subset \operatorname{bd}_{Y_{\alpha}}\left(C_{\alpha}^{\prime}\right) \cup\left(\operatorname{bd}\left(C_{\alpha}\right)-O\right)$. Note that

$$
\begin{gathered}
C_{\alpha}^{\prime}-O=B_{\alpha}^{\prime}-O=C_{\alpha}-O, \\
\operatorname{bd}\left(C_{\alpha}^{\prime}\right)-O \subset \operatorname{bd}\left(C_{\alpha}\right)-O, \\
\operatorname{bd}\left(C_{\alpha}^{\prime}\right) \cap O \subset \operatorname{bd}_{Y_{\alpha}}\left(C_{\alpha}^{\prime}\right) \cap O .
\end{gathered}
$$

Hence $\left(\operatorname{bd}\left(C_{\alpha}^{\prime}\right) \cap O\right) \cap\left(Z_{\alpha} \cup Z\right)=\emptyset$. By the construction, we see that $C_{\alpha}^{\prime}$ satisfies the conditions (1)-(5), and $\mathcal{G}(j) \cup\left\{\operatorname{bd}\left(C_{\beta}^{\prime}\right) \cap O \mid \beta \leq \alpha\right\}$ is in general position for each $j \in \mathbb{N}$.

By transfinite induction on the ordinal $\alpha$, we obtain the desired open shrinking $\mathcal{C}^{\prime}=$ $\left\{C_{\alpha}^{\prime} \mid \alpha<\tau\right\}$ of $\mathcal{C}$. 
Lemma 3.4. (cf. [5, Lemma 2.2]) Let $(X, f)$ be a dynamical system of a metric space $X$ such that $\operatorname{dim} X=n<\infty$ and $\operatorname{dim} P(f) \leq 0$. Let $\mathcal{C}=\left\{C_{\alpha} \mid \alpha<\tau\right\}$ be a locally finite open cover of $X$ and let $\mathcal{B}=\left\{B_{\alpha} \mid \alpha<\tau\right\}$ be a closed shrinking of $\mathcal{C}$. Then for each $k=0,1,2, \ldots$, there is an open shrinking $\mathcal{C}^{\prime}=\left\{C_{\alpha}^{\prime} \mid \alpha<\tau\right\}$ of $\mathcal{C}$ such that for each $\alpha<\tau$,

(1) $B_{\alpha} \subset C_{\alpha}^{\prime} \subset C_{\alpha}$,

(2) $\left\{f^{j}\left(\operatorname{bd}\left(C_{\alpha}^{\prime}\right)\right) \mid \alpha<\tau\right.$ and $\left.|j| \leq k\right\}$ is in general position,

(3) $\operatorname{bd}\left(C_{\alpha}^{\prime}\right) \cap P(f)=\emptyset$.

Proof. We proceed by induction on $k$. In the case $k=0$, if we put $O=X$ and $Z=P(f)$, by Lemma 3.3 there is an open shrinking $\mathcal{C}^{\prime}=\left\{C_{\alpha}^{\prime} \mid \alpha<\tau\right\}$ of $\mathcal{C}$ such that (1)-(3).

Next we suppose the result holds for $k-1$. Then there is an open shrinking $\mathcal{D}=$ $\left\{D_{\alpha} \mid \alpha<\tau\right\}$ of $\mathcal{C}$ such that for each $\alpha<\tau$,

(1') $B_{\alpha} \subset D_{\alpha} \subset C_{\alpha}$

(2') $\left\{f^{j}\left(\operatorname{bd}\left(D_{\alpha}\right)\right) \mid \alpha<\tau\right.$ and $\left.|j| \leq k-1\right\}$ is in general position,

$\left(3^{\prime}\right) \operatorname{bd}\left(D_{\alpha}\right) \cap P(f)=\emptyset$.

Put

$$
F=\bigcup_{\alpha<\tau} \operatorname{bd}\left(D_{\alpha}\right)
$$

Since $F$ contains no periodic points, we can choose a locally finite open (in $X$ ) cover $\mathcal{O}=\left\{O_{\beta} \mid \beta<\gamma\right\}$ of $F$ such that

$$
\left\{f^{-2 k}\left(O_{\beta}\right), \ldots, O_{\beta}, \ldots, f^{2 k}\left(O_{\beta}\right)\right\}
$$

is pairwise disjoint for each $\beta<\gamma$. We may assume that $O_{0}=\emptyset$. By transfinite induction on $\beta$, we will construct an ordered collection $\{\mathcal{D}(\beta) \mid \beta<\gamma\}$ of open shrinkings of $\mathcal{C}$ such that for each $\beta<\gamma$,

(a) $\mathcal{D}(0)=\mathcal{D}$,

(b) $\mathcal{D}(\beta)=\left\{D(\beta)_{\alpha} \mid \alpha<\tau\right\}$,

(c) $B_{\alpha} \subset D(\beta)_{\alpha} \subset \bigcap_{\beta^{\prime}<\beta} D\left(\beta^{\prime}\right)_{\alpha}$,

(d) $\bigcap_{\beta^{\prime}<\beta} D\left(\beta^{\prime}\right)_{\alpha} \cap\left(X-O_{\beta}\right)=D(\beta)_{\alpha} \cap\left(X-O_{\beta}\right)$,

$\operatorname{bd}\left(\bigcap_{\beta^{\prime}<\beta} D\left(\beta^{\prime}\right)_{\alpha}\right) \cap\left(X-O_{\beta}\right) \supset \operatorname{bd}\left(D(\beta)_{\alpha}\right) \cap\left(X-O_{\beta}\right)$, and

if $\operatorname{bd}\left(\bigcap_{\beta^{\prime}<\beta} D\left(\beta^{\prime}\right)_{\alpha}\right) \cap O_{\beta}=\emptyset$, then $\bigcap_{\beta^{\prime}<\beta} D\left(\beta^{\prime}\right)_{\alpha}=D(\beta)_{\alpha}$,

(e) $\mathcal{G}(\beta)=\left\{f^{p}\left(\operatorname{bd}\left(D(\beta)_{\alpha}\right)\right)|| p \mid \leq k-1\right.$ and $\left.\alpha<\tau\right\} \cup\left\{f^{p}\left(\operatorname{bd}\left(D(\beta)_{\alpha}\right) \cap\left(\bigcup_{\beta^{\prime} \leq \beta} O_{\beta^{\prime}}\right)\right) \mid\right.$ $|p|=k$ and $\alpha<\tau\}$ is in general position,

(f) $\operatorname{bd}\left(D(\beta)_{\alpha}\right) \cap P(f)=\emptyset$.

We construct $\mathcal{D}(\beta)$ by transfinite induction on $\beta<\gamma$. For $\beta=0$, we have $\mathcal{D}(0)=\mathcal{D}$. Suppose that we have $\left\{\mathcal{D}\left(\beta^{\prime}\right) \mid \beta^{\prime}<\beta\right\}$ satisfying the desired conditions. To construct $\mathcal{D}(\beta)$, we consider the collection $\mathcal{E}=\left\{E_{\alpha} \mid \alpha<\tau\right\}$, where $E_{\alpha}=\bigcap_{\beta^{\prime}<\beta} D\left(\beta^{\prime}\right)_{\alpha}$. Note that by (d) and local finiteness of $\mathcal{O}$, for each $x \in X$ there is an open neighborhood $U(x)$ of $x$ in $X$ and $\beta_{x}<\beta$ such that $E_{\alpha} \cap U(x)=D\left(\beta_{x}\right)_{\alpha} \cap U(x)$ and $\operatorname{bd}\left(E_{\alpha}\right) \cap U(x)=$ $\operatorname{bd}\left(D\left(\beta_{x}\right)_{\alpha}\right) \cap U(x)$. 
Claim 1. $\mathcal{E}$ is an open shrinking of $\mathcal{C}$ such that $B_{\alpha} \subset E_{\alpha} \subset C_{\alpha}$ and $\operatorname{bd}\left(E_{\alpha}\right) \cap P(f)=\emptyset$.

Proof. Since

$$
E_{\alpha}=\bigcup_{x \in X}\left(D\left(\beta_{x}\right)_{\alpha} \cap U(x)\right) \supset \bigcup_{x \in X}\left(B_{\alpha} \cap U(x)\right)=B_{\alpha},
$$

it follows that $E_{\alpha}$ is open in $X$ and contains $B_{\alpha}$. On the other hand, since

$$
\operatorname{bd}\left(E_{\alpha}\right)=\bigcup_{x \in X}\left(\operatorname{bd}\left(D\left(\beta_{x}\right)_{\alpha}\right) \cap U(x)\right) \text { and } \operatorname{bd}\left(D\left(\beta_{x}\right)_{\alpha}\right) \cap P(f)=\emptyset,
$$

it follows that $\operatorname{bd}\left(E_{\alpha}\right) \cap P(f)=\emptyset$.

Claim 2. $\mathcal{F}=\left\{f^{p}\left(\operatorname{bd}\left(E_{\alpha}\right)\right)|| p \mid \leq k-1\right.$ and $\left.\alpha<\tau\right\} \cup\left\{f^{p}\left(\operatorname{bd}\left(E_{\alpha}\right) \cap\left(\bigcup_{\beta^{\prime}<\beta} O_{\beta^{\prime}}\right)\right)|| p \mid=\right.$ $k$ and $\alpha<\tau\}$ is in general position.

Proof. Note that by (d) and local finiteness of $\bigcup_{|p| \leq k} f^{p}(\mathcal{O})$, for each $x \in X$ there is an open neighborhood $V(x)$ of $x$ in $X$ and $\gamma_{x}<\beta$ such that

$$
f^{p}\left(\operatorname{bd}\left(E_{\alpha}\right)\right) \cap V(x)=f^{p}\left(\operatorname{bd}\left(D\left(\gamma_{x}\right)_{\alpha}\right)\right) \cap V(x)
$$

for each $p$. Choose a locally finite open refinement $\mathcal{W}$ of $\{V(x) \mid x \in X\}$. For each $W \in \mathcal{W}$, we take $x \in X$ with $W \subset V(x)$. Then

$$
\begin{gathered}
f^{p}\left(\operatorname{bd}\left(E_{\alpha}\right)\right) \cap W=f^{p}\left(\operatorname{bd}\left(D\left(\gamma_{x}\right)_{\alpha}\right)\right) \cap W \\
f^{p}\left(\operatorname{bd}\left(E_{\alpha}\right) \cap\left(\bigcup_{\beta^{\prime}<\beta} O_{\beta^{\prime}}\right)\right) \cap W=f^{p}\left(\operatorname{bd}\left(D\left(\gamma_{x}\right)_{\alpha}\right) \cap\left(\bigcup_{\beta^{\prime} \leq \gamma_{x}} O_{\beta^{\prime}}\right)\right) \cap W .
\end{gathered}
$$

By (e), $\left.\mathcal{F}\right|_{W}=\left.\mathcal{G}\left(\gamma_{x}\right)\right|_{W}$ is in general position. Now we will show that $\mathcal{F}$ is in general position. Let $\mathcal{S} \subset \mathcal{F}$ such that $1 \leq|\mathcal{S}| \leq n+1$ and $\bigcap \mathcal{S} \neq \emptyset$. Note that $(\bigcap \mathcal{S}) \cap W$ is an at most $(n-|\mathcal{S}|)$-dimensional $F_{\sigma}$-set of $X$. By local finiteness of $\mathcal{W}$,

$$
\bigcap \mathcal{S}=\bigcup_{W \in \mathcal{W}}((\bigcap \mathcal{S}) \cap W)
$$

is also an at most $(n-|\mathcal{S}|)$-dimensional.

Then for each $|q| \leq k$,

$$
\begin{aligned}
\mathcal{S}_{q}=\left\{f^{p}\left(\operatorname{bd}\left(E_{\alpha}\right)\right) \cap f^{q}\left(O_{\beta}\right) \mid\right. & |p| \leq k-1 \text { and } \alpha<\tau\} \\
& \cup\left\{f^{p}\left(\operatorname{bd}\left(E_{\alpha}\right) \cap \bigcup_{\beta^{\prime}<\beta} O_{\beta^{\prime}}\right) \cap f^{q}\left(O_{\beta}\right)|| p \mid=k \text { and } \alpha<\tau\right\}
\end{aligned}
$$

is also in general position in $f^{q}\left(O_{\beta}\right)$. Thus, $f^{-q}\left(\mathcal{S}_{q}\right)$ is also in general position in $O_{\beta}$. By Lemma 3.3 and Claim 1, there is an open shrinking $\mathcal{D}(\beta)$ of $\left\{E_{\alpha} \mid \alpha<\tau\right\}(=$ 
$\left.\left\{\bigcap_{\beta^{\prime}<\beta} D\left(\beta^{\prime}\right)_{\alpha} \mid \alpha<\tau\right\}\right)$ such that

(1') $B_{\alpha} \subset D(\beta)_{\alpha} \subset \bigcap_{\beta^{\prime}<\beta} D\left(\beta^{\prime}\right)_{\alpha}\left(=E_{\alpha}\right)$,

(2') $D(\beta)_{\alpha}=\bigcap_{\beta^{\prime}<\beta} D\left(\beta^{\prime}\right)_{\alpha}$ if bd $\bigcap_{\beta^{\prime}<\beta}\left(D\left(\beta^{\prime}\right)_{\alpha}\right) \cap O_{\beta}=\emptyset$,

(3') $D(\beta)_{\alpha}-O_{\beta}=\bigcap_{\beta^{\prime}<\beta} D\left(\beta^{\prime}\right)_{\alpha}-O_{\beta}$,

(4') $\operatorname{bd}\left(D(\beta)_{\alpha}\right)-O_{\beta} \subset \operatorname{bd}\left(\bigcap_{\beta^{\prime}<\beta} D\left(\beta^{\prime}\right)_{\alpha}\right)-O_{\beta}$,

$\left(5^{\prime}\right) \operatorname{bd}\left(D(\beta)_{\alpha}\right) \cap P(f)=\emptyset$

(6') $f^{-q}\left(\mathcal{S}_{q}\right) \cup\left\{\operatorname{bd}\left(D(\beta)_{\alpha}\right) \cap O_{\beta} \mid \alpha<\tau\right\}$ is in general position for each $|q| \leq k$.

By the similar arguments to the proof of [7, Lemma 3.5], we can check (e). By (4') and Claim 2, it suffices to show that $\left.\mathcal{G}(\beta)\right|_{f^{q}\left(O_{\beta}\right)}$ is in general position for each $q$. Note that by (6'), for $|p| \leq k$,

$$
\mathcal{S}_{q} \cup\left\{f^{q}\left(\operatorname{bd}\left(D(\beta)_{\alpha}\right) \cap f^{q}\left(O_{\beta}\right)\right) \mid \alpha<\tau\right\}
$$

is in general position in $f^{q}\left(O_{\beta}\right)$. Our goal is to see that all elements of $\left.\mathcal{G}(\beta)\right|_{f^{q}\left(O_{\beta}\right)}$ are contained in distinct elements of $\mathcal{S}_{q} \cup\left\{f^{q}\left(\operatorname{bd}\left(D(\beta)_{\alpha}\right) \cap f^{q}\left(O_{\beta}\right)\right) \mid \alpha<\tau\right\}$. It suffices to prove the following claim.

Claim 3. Let $\alpha<\tau$ and $|p| \leq k$ with $p \neq q$.

(i) If $|p| \leq k-1$, then

$$
f^{p}\left(\operatorname{bd}\left(D(\beta)_{\alpha}\right)\right) \cap f^{q}\left(O_{\beta}\right) \subset f^{p}\left(\operatorname{bd}\left(\bigcap_{\beta^{\prime}<\beta} D\left(\beta^{\prime}\right)_{\alpha}\right)\right) \cap f^{q}\left(O_{\beta}\right) .
$$

(ii) If $|p|=k$, then

$$
f^{p}\left(\operatorname{bd}\left(D(\beta)_{\alpha}\right) \cap \bigcup_{\beta^{\prime} \leq \beta} O_{\beta^{\prime}}\right) \cap f^{q}\left(O_{\beta}\right) \subset f^{p}\left(\operatorname{bd}\left(\bigcap_{\beta^{\prime}<\beta} D\left(\beta^{\prime}\right)_{\alpha}\right) \cap \bigcup_{\beta^{\prime}<\beta} O_{\beta^{\prime}}\right) \cap f^{q}\left(O_{\beta}\right) .
$$

Proof. Note that $f^{q}\left(O_{\beta}\right) \cap f^{p}\left(O_{\beta}\right)=\emptyset$. If $|p| \leq k-1$, then

$$
\begin{aligned}
f^{p}\left(\operatorname{bd}\left(D(\beta)_{\alpha}\right)\right) \cap f^{q}\left(O_{\beta}\right) & =f^{p}\left(\operatorname{bd}\left(D(\beta)_{\alpha}\right)-O_{\beta}\right) \cap f^{q}\left(O_{\beta}\right) \\
& \subset f^{p}\left(\operatorname{bd}\left(\bigcap_{\beta^{\prime}<\beta} D\left(\beta^{\prime}\right)_{\alpha}\right)-O_{\beta}\right) \cap f^{q}\left(O_{\beta}\right) \\
& =f^{p}\left(\operatorname{bd}\left(\bigcap_{\beta^{\prime}<\beta} D\left(\beta^{\prime}\right)_{\alpha}\right)\right) \cap f^{q}\left(O_{\beta}\right),
\end{aligned}
$$

On the other hand, if $|p|=k$, then

$$
\begin{aligned}
f^{p}\left(\operatorname{bd}\left(D(\beta)_{\alpha}\right) \cap \bigcup_{\beta^{\prime} \leq \beta} O_{\beta^{\prime}}\right) \cap f^{q}\left(O_{\beta}\right) & =f^{p}\left(\operatorname{bd}\left(D(\beta)_{\alpha}\right) \cap\left[\bigcup_{\beta^{\prime} \leq \beta} O_{\beta^{\prime}}-O_{\beta}\right]\right) \cap f^{q}\left(O_{\beta}\right) \\
& \subset f^{p}\left(\operatorname{bd}\left(\bigcap_{\beta^{\prime}<\beta} D\left(\beta^{\prime}\right)_{\alpha}\right) \cap\left[\bigcup_{\beta^{\prime}<\beta} O_{\beta^{\prime}}-O_{\beta}\right]\right) \cap f^{q}\left(O_{\beta}\right) \\
& =f^{p}\left(\operatorname{bd}\left(\bigcap_{\beta^{\prime}<\beta} D\left(\beta^{\prime}\right)_{\alpha}\right) \cap \bigcup_{\beta^{\prime}<\beta} O_{\beta^{\prime}}\right) \cap f^{q}\left(O_{\beta}\right)
\end{aligned}
$$


Therefore, by induction on $\beta$ we obtain the desired collection $\{\mathcal{D}(\beta) \mid \beta<\gamma\}$ of open shrinkings of $\mathcal{C}$.

Finally, we put $C_{\alpha}^{\prime}=\bigcap_{\beta<\gamma} D(\beta)_{\alpha}$ for each $\alpha<\tau$. By the arguments of Claim 1 and 2, we see that $\mathcal{C}^{\prime}=\left\{C_{\alpha}^{\prime} \mid \alpha<\tau\right\}$ is an open shrinking of $\mathcal{C}$ satisfying the conditions (1)-(3).

Lemma 3.5. ([7, Lemma 3.7]) Let $(X, f)$ be a dynamical system of a metric space $X$ such that $\operatorname{dim} X=n<\infty$ and $\operatorname{dim} P(f) \leq 0$. Then, for each $j \in \mathbb{N}$, there is a locally finite open cover $\mathcal{C}(j)=\left\{C(j)_{\alpha} \mid \alpha<\tau\right\}$ of $X$ such that

(1) $\operatorname{mesh}(\mathcal{C}(j))<1 / j$,

$(2) \operatorname{ord}(\mathcal{G}) \leq n$, where $\mathcal{G}=\left\{f^{p}\left(\operatorname{bd}\left(C(j)_{\alpha}\right)\right) \mid \alpha<\tau, j \in \mathbb{N}\right.$ and $\left.p \in \mathbb{Z}\right\}$.

Proof. For each $j \in \mathbb{N}$, we choose a locally finite open cover $\mathcal{D}(j)$ of $X$ such that $\operatorname{mesh}(\mathcal{D}(j))<1 / j$. For simplicity, we may assume that $\mathcal{D}(j)=\left\{D(j)_{\alpha} \mid \alpha<\tau\right\}$ by use of some sufficiently large ordinal number $\tau$. Note that $D(j)_{\alpha}$ may be an empty set. Take an open shrinking $\mathcal{B}(j)=\left\{B(j)_{\alpha} \mid \alpha<\tau\right\}$ of $\mathcal{D}(j)$ such that $\overline{\mathcal{B}}(j)=\left\{\operatorname{cl}\left(B(j)_{\alpha}\right) \mid \alpha<\tau\right\}$ is a closed shrinking of $\mathcal{D}(j)$. For each $j \in \mathbb{N}$ and each $k \in \mathbb{N}$ with $k \geq j$, we will find an open shrinking $\mathcal{D}(j, k)=\left\{D(j, k)_{\alpha} \mid \alpha<\tau\right\}(k \geq j)$ of $\mathcal{D}(j)$ and a closed shrinking $\mathcal{B}(j, k)=\left\{B(j, k)_{\alpha} \mid \alpha<\tau\right\}(k \geq j)$ of $\mathcal{D}(j, k)$ such that

(a) $\mathcal{D}(j, j)=\mathcal{D}(j), \mathcal{B}(j, j)=\overline{\mathcal{B}}(j)$

(b) $\operatorname{cl}\left(B(j)_{\alpha}\right)=B(j, j)_{\alpha} \subset B(j, j+1)_{\alpha} \subset \cdots \subset D(j, j+1)_{\alpha} \subset D(j, j)_{\alpha}=D(j)_{\alpha}$,

(c) $\operatorname{ord}\left\{\operatorname{cl}\left(f^{p}\left(D(j, k+1)_{\alpha}-B(j, k+1)_{\alpha}\right)\right) \mid \alpha<\tau, 1 \leq j \leq k\right.$ and $\left.|p| \leq k\right\} \leq n$.

We proceed by induction on $k$. Suppose that we have

$$
\mathcal{D}(1, k), \ldots, \mathcal{D}(k-1, k), \mathcal{D}(k, k)=\mathcal{D}(k)
$$

and

$$
\mathcal{B}(1, k), \ldots, \mathcal{B}(k-1, k), \mathcal{B}(k, k)=\overline{\mathcal{B}}(k)
$$

satisfying the desired conditions. We will construct $\mathcal{D}(1, k+1), \ldots, \mathcal{D}(k, k+1)$ and $\mathcal{B}(1, k+1), \ldots, \mathcal{B}(k, k+1)$. Note that $\left\{D(j, k)_{\alpha} \mid 1 \leq j \leq k\right.$ and $\left.\alpha<\tau\right\}$ is a locally finite open cover of $X$ and $\left\{B(j, k)_{\alpha} \mid 1 \leq j \leq k\right.$ and $\left.\alpha<\tau\right\}$ is a closed shrinking of $\left\{D(j, k)_{\alpha} \mid 1 \leq j \leq k\right.$ and $\left.\alpha<\tau\right\}$. By Lemma 3.4, there is an open shrinking $\left\{D(j, k+1)_{\alpha} \mid 1 \leq j \leq k\right.$ and $\left.\alpha<\tau\right\}$ of $\left\{D(j, k)_{\alpha} \mid 1 \leq j \leq k\right.$ and $\left.\alpha<\tau\right\}$ such that

(1') $B(j, k)_{\alpha} \subset D(j, k+1)_{\alpha} \subset D(j, k)_{\alpha}$,

(2') $\left\{f^{p}\left(\operatorname{bd}\left(D(j, k+1)_{\alpha}\right)\right) \mid \alpha<\tau, 1 \leq j \leq k\right.$ and $\left.|p| \leq k\right\}$ is in general position.

Since

$$
\left\{f^{p}\left(\operatorname{bd}\left(D(j, k+1)_{\alpha}\right)\right) \mid \alpha<\tau, 1 \leq j \leq k \text { and }|p| \leq k\right\}
$$

is a locally finite collection of closed subsets of $X$ with ord $\leq n$, there is an open swelling

$$
\mathcal{O}(j, k+1)=\left\{O(j, k+1)_{\alpha} \mid \alpha<\tau\right\}
$$

of $\left\{\operatorname{bd}\left(D(j, k+1)_{\alpha}\right) \mid \alpha<\tau\right\}$ such that

(3') $O(j, k+1)_{\alpha} \cap B(j, k)_{\alpha}=\emptyset$, 
(4') $\operatorname{ord}\left\{f^{p}\left(\operatorname{cl}\left(O(j, k+1)_{\alpha}\right)\right) \mid \alpha<\tau, 1 \leq j \leq k\right.$ and $\left.|p| \leq k\right\} \leq n$.

For each $\alpha<\tau$ and $j \in \mathbb{N}$, let

$$
B(j, k+1)_{\alpha}=\operatorname{cl}\left(D(j, k+1)_{\alpha}\right)-O(j, k+1)_{\alpha} .
$$

Then $\mathcal{D}(j, k+1)$ and $\mathcal{B}(j, k+1)$ satisfy the conditions (a)-(c).

Now put

$$
C(j)_{\alpha}=\operatorname{int}\left[\bigcap_{k=j}^{\infty} D(j, k)_{\alpha}\right] \text { and } \mathcal{C}(j)=\left\{C(j)_{\alpha} \mid \alpha<\tau\right\} .
$$

Note that $B(j)_{\alpha} \subset C(j)_{\alpha} \subset D(j)_{\alpha}$. Since (1) is obvious, we only need to check (2). It suffices to show that for each $k \in \mathbb{N}$,

$$
\mathcal{G}(k)=\left\{f^{p}\left(\operatorname{bd}\left(C(j)_{\alpha}\right)\right) \mid \alpha<\tau, 1 \leq j \leq k \text { and }|p| \leq k\right\}
$$

is ord $\leq n$. However, since

$$
\begin{gathered}
\operatorname{bd}\left(C(j)_{\alpha}\right) \subset \operatorname{cl}\left(D(j, k+1)_{\alpha}-B(j, k+1)_{\alpha}\right) \text { and } \\
\operatorname{ord}\left\{\operatorname{cl}\left(f^{p}\left(D(j, k+1)_{\alpha}-B(j, k+1)_{\alpha}\right)\right) \mid \alpha<\tau, 1 \leq j \leq k \text { and }|p| \leq k\right\} \leq n,
\end{gathered}
$$

we see that (2) holds. Consequently, we obtain the desired open cover $\mathcal{C}(j)=\left\{C(j)_{\alpha} \mid\right.$ $\alpha<\tau\}$ of $X$ for each $j \in \mathbb{N}$.

Lemma 3.6. (cf. [7, Lemma 3.8]) Let $(X, f)$ be a dynamical system of a metric space $X$ with $\operatorname{dim} X=n<\infty$. Suppose that for each $j \in \mathbb{N}, \mathcal{C}(j)=\left\{C(j)_{\alpha} \mid \alpha<\tau\right\}$ is a locally finite open cover of $X$ such that $\operatorname{mesh}(\mathcal{C}(j))<1 / j$ for each $j>0$ and $\operatorname{ord}(\mathcal{G}) \leq n$, where $\mathcal{G}=\left\{f^{p}\left(\operatorname{bd}\left(C(j)_{\alpha}\right)\right) \mid \alpha<\tau, j \in \mathbb{N}\right.$ and $\left.p \in \mathbb{Z}\right\}$. Then, for each $j \in \mathbb{N}$ there is a locally finite regular closed partition $\mathcal{D}(j)$ of $X$ such that

(1) $\operatorname{mesh}(\mathcal{D}(j)) \leq 1 / j$

(2) $\operatorname{ord}(\mathcal{D}(j)) \leq 2^{n}$,

(3) $\mathcal{D}(j+1)$ is a refinement of $f^{-1}(\mathcal{D}(j)) @ \mathcal{D}(j) @ f(\mathcal{D}(j))$.

Proof. Put $C^{\prime}(j)_{0}=\operatorname{cl}\left(C(j)_{0}\right)$ and $C^{\prime}(j)_{\alpha}=\operatorname{cl}\left(\operatorname{int}\left(C(j)_{\alpha}-\left(\bigcup_{\alpha^{\prime}<\alpha} C(j)_{\alpha^{\prime}}\right)\right)\right)$. Then $\mathcal{C}^{\prime}(j)=$ $\left\{C^{\prime}(j)_{\alpha} \mid \alpha<\tau\right\}$ is a locally finite regular closed partition of $X$. Let

$$
\mathcal{D}(j)=@_{0 \leq i \leq j,|p| \leq j} f^{p}\left(\mathcal{C}^{\prime}(i)\right) .
$$

We will show that $\mathcal{D}(j)$ is a desired partition. Since (1) and (3) are obvious, we only need to check (2).

Let $x \in X$. For each $j \in \mathbb{N}$ and $p \in \mathbb{Z}$, put

$$
\left.m_{j, p}=\operatorname{ord}_{f^{p}(x)}\left\{\operatorname{bd}\left(C(j)_{\alpha}\right)\right) \mid \alpha<\tau\right\} .
$$


Since $\operatorname{ord}_{x} \mathcal{G} \leq n$, we have

$$
\sum_{\substack{j \in \mathbb{N} \\ p \in \mathbb{Z}}} m_{j, p} \leq n .
$$

We will show that $\operatorname{ord}_{f^{p}(x)} \mathcal{C}^{\prime}(j) \leq m_{j, p}+1$. Put $\alpha_{0}=\min \left\{\alpha<\tau \mid f^{p}(x) \in C(j)_{\alpha}\right\}$. By [1, Lemma 13], we can see the following;

$$
\begin{aligned}
\operatorname{ord}_{f^{p}(x)} \mathcal{C}^{\prime}(j) & =\operatorname{ord}_{f^{p}(x)}\left\{C^{\prime}(j)_{\alpha} \mid \alpha<\alpha_{0}\right\} \cup\left\{C^{\prime}(j)_{\alpha_{0}}\right\} \cup\left\{C^{\prime}(j)_{\alpha} \mid \alpha>\alpha_{0}\right\} \\
& \leq \operatorname{ord}_{f^{p}(x)}\left\{\operatorname{cl}\left(C(j)_{\alpha}\right)-C(j)_{\alpha} \mid \alpha<\alpha_{0}\right\} \cup\left\{C^{\prime}(j)_{\alpha_{0}}\right\} \\
& \leq m_{j, p}+1
\end{aligned}
$$

Note that $\operatorname{ord}(\mathcal{A} @ \mathcal{B}) \leq \operatorname{ord}(\mathcal{A}) \cdot \operatorname{ord}(\mathcal{B})$ for each regular closed partitions $\mathcal{A}$ and $\mathcal{B}$ of $X$. Since $m+1 \leq 2^{m}$ for each $m=0,1,2, \ldots$,

$$
\begin{aligned}
\operatorname{ord}_{x} \mathcal{D}(j) & =\operatorname{ord}_{\substack { x \\
\begin{subarray}{c}{0 \leq i \leq j \\
|p| \leq j{ x \\
\begin{subarray} { c } { 0 \leq i \leq j \\
| p | \leq j } }\end{subarray}} f^{p}\left(\mathcal{C}^{\prime}(i)\right) \leq \prod_{\substack{0 \leq i \leq j \\
|p| \leq j}} \operatorname{ord}_{x} f^{-p}\left(\mathcal{C}^{\prime}(i)\right)=\prod_{\substack{0 \leq i \leq j \\
|p| \leq j}} \operatorname{ord}_{f^{p}(x)} \mathcal{C}^{\prime}(i) \\
& \leq \prod_{\substack{0 \leq i \leq j \\
|p| \leq j}}\left(m_{i, p}+1\right) \leq \prod_{\substack{0 \leq i \leq j \\
|p| \leq j}} 2^{m_{i, p}}=2^{\sum m_{i, p}} \leq 2^{n} .
\end{aligned}
$$

Therefore, $\mathcal{D}(j)$ is a desired partition.

Finally, we prove our main theorem in this paper.

Proof of Theorem 3.1. By Lemma 3.5, we have a sequence of locally finite open covers of $X$ satisfying the conditions of Lemma 3.6. Applying Lemma 3.6, we have regular closed partitions satisfying the conditions of Lemma 2.1. Hence, by Lemma 2.1, there is a homeomorphism $\tilde{f}: C \rightarrow C$ of a zero-dimensional metric space $C$ and an at most $2^{n}$-to-one closed onto map $p: C \rightarrow X$ such that $p \circ \tilde{f}=f \circ p$.

Remark 2. In the statements of Theorem 3.1, the following are also true:

(1) The $2^{n}$-to-one closed onto map $p$ satisfies the condition that $\left|p^{-1}(x)\right|=1$ for any $x \in P(f)$, i.e. $P(f)$ is naturally embedded in $C$.

(2) If $X$ is a complete metric space, then $C$ can be taken as a complete metric space and there is a $G_{\delta}$-dense set $G$ of $X$ such that $\left|p^{-1}(x)\right|=1$ for each $x \in G$. In fact, in the proof of Lemma 3.6, consider the set $G=X-\bigcup_{j \in \mathbb{N}}[\bigcup\{\operatorname{bd}(D) \mid D \in \mathcal{D}(j)\}]$. Then $G$ satisfies the desired condition.

(3) If $X$ is a perfect space, then $C$ can be taken as a perfect space.

Finally, as a special case we consider the case that $f: X \rightarrow X$ is an expansive homeomorphism of a compact metric space $X$. A homeomorphism $f: X \rightarrow X$ of a compact metric space $(X, d)$ is expansive if there is $\epsilon>0$ such that for any $x, y \in X$ with $x \neq y$, there is an integer $k \in \mathbb{Z}$ such that $d\left(f^{k}(x), f^{k}(y)\right) \geq \epsilon$. Such an $\epsilon>0$ is called an expansive constant for $f$. In [10], Mañé proved that if a compact metric space $X$ admits 
an expansive homeomorphism $f$ on $X$, then $\operatorname{dim} X<\infty$ and every minimal set of $f$ is zero-dimensional. Moreover, we see that $\operatorname{dim} I_{0}(f) \leq 0$, where

$$
I_{0}(f)=\bigcup\{M \mid M \text { is a zero-dimensional } f \text {-invariant closed set of } X\}
$$

(see [6, Proposition 2.5]). Let $Y_{k}=\{1,2, \ldots, k\} \quad(k \in \mathbb{N})$ be the discrete space having $k$-elements and let $Y_{k}^{\mathbb{Z}}=\prod_{-\infty}^{\infty} Y_{k}$ be the product space. Then the shift homeomorphism $\sigma: Y_{k}^{\mathbb{Z}} \rightarrow Y_{k}^{\mathbb{Z}}$ is defined by $\sigma\left(\left(x_{j}\right)_{j}\right)=\left(x_{j+1}\right)_{j}$.

Corollary 3.7. (cf. [7, p. 950]) Let $f: X \rightarrow X$ be an expansive homeomorphism of a compact metric space $X$ with $\operatorname{dim} X=n$. Then there exist $k \in \mathbb{N}$, a closed $\sigma$-invariant set $\Sigma$ of $\sigma: Y_{k}^{\mathbb{Z}} \rightarrow Y_{k}^{\mathbb{Z}}$ and an at most $2^{n}$-to-one onto map $p: \Sigma \rightarrow X$ such that $p \circ \sigma=f \circ p$ and $\left|p^{-1}(x)\right|=1$ for any $x \in I_{0}(f)$.

Proof. Let $\epsilon>0$ be an expansive constant for $f$. Since $\operatorname{dim} I_{0}(f) \leq 0$, by Lemma 3.5 there is a finite open cover $\mathcal{C}(\epsilon)$ of $X$ such that

(1) $\operatorname{mesh}(\mathcal{C}(\epsilon))<\epsilon$

$(2) \operatorname{ord}(\mathcal{G}) \leq n$, where $\mathcal{G}=\left\{f^{j}(\operatorname{bd}(C)) \mid C \in \mathcal{C}(\epsilon), j \in \mathbb{Z}\right\}$,

(3) $\operatorname{bd}(C) \cap I_{0}(f)=\phi$ for each $C \in \mathcal{C}(\epsilon)$.

Let $\mathcal{C}(\epsilon)=\left\{C_{1}, C_{2}, \ldots, C_{k}\right\}$ and put

$$
C_{1}^{\prime}=\operatorname{cl}\left(C_{1}\right), C_{i}^{\prime}=\operatorname{cl}\left(C_{i}-\left(C_{1} \cup C_{2} \cup \ldots \cup C_{i-1}\right)\right)(i \geq 2) .
$$

Consider the set

$$
\Sigma=\left\{\left(i_{j}\right)_{j} \in Y_{k}^{\mathbb{Z}} \mid \bigcap_{j \in \mathbb{Z}} f^{-j}\left(C_{i_{j}}^{\prime}\right) \neq \phi\right\}
$$

Note that $\bigcap_{j \in \mathbb{Z}} f^{-j}\left(C_{i_{j}}^{\prime}\right)$ is a one point set for each $\left(i_{j}\right)_{j} \in \Sigma$, because $f$ is expansive. Also, we see that $\sigma(\Sigma)=\Sigma$. Define a map $p: \Sigma \rightarrow X$ by

$$
p\left(\left(i_{j}\right)_{j}\right)=\bigcap_{j \in \mathbb{Z}} f^{-j}\left(C_{i_{j}}^{\prime}\right)
$$

By the proof of Lemma 3.6, we see that $p: \Sigma \rightarrow X$ is a desired map.

Acknowledgment. The authors would like to thank the referee for helpful comments.

\section{References}

[1] J. M. Arts, R. J. Fokkink and H. Vermeer, Variations on a theorem of Lusternik and Schnirelmann, Topology, 35 (1996), 1051-1056.

[2] J. M. Arts, R. J. Fokkink and J. Vermeer, A dynamical decomposition theorem, Acta Math. Hung., 94(3), 2002, 191-196. 
[3] R. Engelking, Theory of Dimensions Finite and Infinite, Heldermann Verlag, Lemgo, 1995.

[4] W. Hurewicz, Ein Theorem der Dimensionstheorie, Ann. of Math. 31 (1930), 176-180.

[5] Y. Ikegami, H. Kato and A. Ueda, Eventual colorings of homeomorphisms, J. Math. Soc. Japan, to appear.

[6] H. Kato, Minimal sets and chaos in the sense of Devaney on continuum-wise expansive homeomorphisms, Lecture Notes in Pure and Appl. Math., 170, Dekker, New York, 1995.

[7] J. Kulesza, Zero-dimensional covers of finite dimensional dynamical systems, Ergod. Th. Dynam. Sys. 15 (1995), 939-950.

[8] K. Kuratowski, Sur l'application des espaces fonctionnels a la Theorie de la dimension, Fundamenta Mathematicae 18 (1932), 285-292.

[9] L. Lusternik and L. Schnirelman, Topological methods in variational calculus (Russian), Moscow, 1930.

[10] R. Mañé, Expansive homeomorphisms and topological dimension, Trans. Amer. Math. Soc. 252 (1979), 313-319.

[11] J. van Mill, The Infinite-Dimensional Topology of Function Spaces, North-Holland publishing Co., Amsterdam, 2001.

[12] K. Morita, A condition for the metrizability of topological spaces and for $n$ dimensionality, Sci. Rep. Tokyo Kyoiku Daigaku, Ser. A 5 (1955), 33-36.

[13] J. Nagata, Modern Dimension Theory, North-Holland publishing Co., Amsterdam, 1965.

Yuki Ikegami

Institute of Mathematics

University of Tsukuba

Ibaraki, 305-8571 Japan

Hisao Kato

Institute of Mathematics

University of Tsukuba

Ibaraki, 305-8571 Japan

e-mail: hkato@math.tsukuba.ac.jp

Akihide Ueda

Institute of Mathematics 
University of Tsukuba

Ibaraki, 305-8571 Japan 